\title{
Comparative analyses of response surface methodology and artificial neural networks on incorporating tetracaine into liposomes
}

\author{
Ana Karina Vidal Pereira ${ }^{1 \#}$, Raquel de Melo Barbosa ${ }^{\circledR 2,3,5^{* \#}}$, Marcelo Augusto Costa Fernandes ${ }^{\circledR 4,6}$, \\ Leandro Finkler ${ }^{1}$, Christine Lamenha Luna Finkler ${ }^{1}$
}

\begin{abstract}
${ }^{1}$ Federal University of Pernambuco, Academic Centre of Vitória, Vitória de Santo Antão, PE, Brazil, ${ }^{2}$ Department of Pharmacy, UNINASSAU College, Natal, RN, Brazil, ${ }^{3}$ Department of Pharmacy, Federal University of Rio Grande do Norte, Natal, RN, Brazil, ${ }^{4}$ Department of Computer Engineering and Automation, Federal University of Rio Grande do Norte, Natal, RN, Brazil, ${ }^{5}$ Current affiliation: MIT Department of Chemical Engineering, Massachusetts Institute of Technology, Cambridge,

MA, USA, 'John A. Paulson School of Engineering and Applied Sciences, Harvard University, Cambridge, MA, USA
\end{abstract}

\begin{abstract}
This study evaluated the incorporation of tetracaine into liposomes by RSM (Response Surface Methodology) and ANN (Artificial Neural Networks) based models. RCCD (rotational central composite design) and ANN were performed to optimize the sonication conditions of particles containing $100 \%$ lipid. Laser light scattering was used to perform measure hydrodynamic radius and size distribution of vesicles. The liposomal formulations were analyzed by incorporating the drug into the hydrophilic phase or the lipophilic phase. RCCD and ANN were conducted, having the lipid/cholesterol ratio and concentration of tetracaine as variables investigated and, the encapsulation efficiency and mean diameter of the vesicles as response variables. The optimum sonication condition set at a power of $16 \mathrm{kHz}$ and 3 minutes, resulting in sizes smaller than $800 \mathrm{~nm}$. Maximum encapsulation efficiency (39.7\%) was obtained in the hydrophilic phase to a tetracaine concentration of $8.37 \mathrm{mg} / \mathrm{mL}$ and $79.5: 20.5 \% \mathrm{lipid} /$ cholesterol ratio. Liposomes were stable for about 30 days (at $4{ }^{\circ} \mathrm{C}$ ), and the drug encapsulation efficiency was higher in the hydrophilic phase. The experimental results of RCCD-RSM and ANN techniques show ANN obtained more refined prediction errors that RCCD-RSM technique, therefore, ANN can be considered as an efficient mathematical method to characterize the incorporation of tetracaine into liposomes.
\end{abstract}

Keywords: Local anesthetics. Liposomes. Response Surface Methodology. Artificial Neural Networks.

\section{INTRODUCTION}

The mathematical modeling of drugs encapsulation has been a research target in the recent years. Based in the mathematical modeling, the researchers can perform estimates about the parameters and minimize the laboratory experiments. There are many techniques can be used for mathematical modeling of drugs encapsulation (Siepmann, Siepmann, 2008). However, methods based on the Response Surface Methodology (RSM) and Artificial Neural Networks (ANN) have been showing satisfactory results (Sun et al., 2003).

The use of statistical experimental planning and RSM explores the relationships between several explanatory variables and one or more response variables

\footnotetext{
*Correspondence: R. M. Barbosa. Departamento de Farmácia, UNINASSAU. Avenida Engenheiro Roberto Freire 1514, Capim Macio, ZP 59082-095, Natal, RN - Brazil. Phone: 5584 99612-4869. E-mail: m.g.barbosafernandes@gmail.com. \#These authors contributed equally to this work.
}

and is one of the most popular methods in the optimization of drug delivery systems. The optimization of variables by RSM includes statistical experimental designs, multiple regression analysis, and mathematical optimization algorithms, used to select the optimum formulation (Subramanian, Yajnik, Murthy, 2004). However, in general, the predictions of response variables are done by a second-order equation. Since this prediction is often limited to low levels, ANN was incorporated to overcome this limitation (Takayama, Fujikawa, Nagai, 1991; Sulaiman et al., 2017; Moussa et al. 2017).

Prediction techniques based on Artificial Intelligence (AI) has been used to characterize formulations and biotechnological processes. Among the AI techniques can highlight the experimental prediction models represented by ANN (Takayama et al., 2003; Subramanian, Yajnik, Murthy, 2004; Zhang et al., 2010). The ANN is generic mathematical models inspired by biological neurons, and other actions work with interpolation (or predictors) universal. These 
structures can learn and can help without any experimental model from a set of data called training data (Haykin, 1998).

Recent researches have been published comparing the data analysis techniques RCCD- RSM and ANN (Desai et al., 2008; Asiltürk, Çunkaş, 2011; Moghaddam, Khajeh, 2011; Ahmadi, Golian, 2011; Maran, Priya, 2015). According to the most of articles presented, the ANN is an alternative better than the methods based on RSM in the case of performance. Furthermore, ANN can increase the level of certainty associated with the results and simultaneously can be used to validation new technological strategies.

The liposomes containing local anesthetic tetracaine, and carriers were prepared in two stages: a hydrophilic phase by incorporating the drug during hydration of the lipid film and a lipophilic phase, incorporating tetracaine during the film preparation. The two forms of preparation were carried out to determine which mathematical modeling approach could result in greater drug encapsulation. In both, the sonication parameters (frequency and duration) were fixed, and the components were changed.

The experiments were performed using an experimental design since there is a substantial lack of application of this statistical tool in research involving systems of controlled drug release. In both cases, RCCD experimental designs and ANN were conducted, having as variables investigated the lipid/cholesterol ratio and the concentration of tetracaine and, encapsulation efficiency and the average size of liposomes as response variables.

The objective of this study was to analyze two different approaches (RSM and ANN) of the mathematical modeling applied to the incorporating tetracaine into liposomes and compare their results. There have been many works in the literature about the encapsulating tetracaine in liposomes (Friedmann, Juliano, 1984; Foldvari, Jarvis, Oguejiofor, 1993; Fisher et al., 1994; El Maghraby, Arafa, Essa, 2018). However, there have been fewer studies about the different mathematical modeling approaches and comparative analyzes. The mathematical modeling studies are necessary to understand the incorporation process and show the optimization alternatives to the process.

\section{MATERIAL AND METHODS}

\section{Material}

Liposomes containing tetracaine (TCC) were prepared using the following reagents: hydrogenated soy phosphatidylcholine (via Farma ${ }^{\circledR}$, São Paulo, Brazil), tetracaine (Sigma-Aldrich Co., St. Louis ${ }^{\circledR}$, MO, USA), chloroform (Merk ${ }^{\circledR}$, Darmstadt, Germany), methanol
(Merk ${ }^{\circledR}$, Darmstadt, Germany) and cholesterol (SigmaAldrich Co., St. Louis ${ }^{\circledR}$, MO, USA). All other chemicals were of analytical grade.

\section{Methods}

\section{Preparation of liposomes}

The preparation of liposomes was performed by the method of Bangham (New, 1990) by rehydrating the dried film with the subsequent homogenization of vesicles size (Lasic, 1993). The phospholipid stored at $-20^{\circ} \mathrm{C}$ was weighed according to the desired composition and then solubilized with cholesterol in $10 \mathrm{~mL}$ of a 9:1 chloroform/ methanol solution $(\mathrm{v} / \mathrm{v})$. The solution was homogenized for 5 minutes in a rotary evaporator, followed by solvent evaporation under vacuum $(650 \mathrm{mmHg})$ at a temperature above the lipid phase transition $\left(\mathrm{Tt}=33^{\circ} \mathrm{C}\right)$ until the dry film formation. In the step of dry film hydration was added $10 \mathrm{~mL}$ of a $60: 40$ ethanol/water solution $(\%, \mathrm{v} / \mathrm{v})$ at room temperature.

Initially, liposomes containing 100\% lipid ( $0.05 \mathrm{~g}$ lipid/10 mL solvent) were added in order to optimize the sonication conditions. This was evaluated from a RCCD experimental design (rotational central composite design) with repetition in triplicate at the central point (level 0 ) and four axial points (levels $\pm \alpha$, where $\alpha=1.41$ ), totaling 11 trials. The independent variables were time and sonication power and the dependent variable (response) was liposome size. The experiments were performed randomly and TABLE I show the coded levels and actual values of the variables.

After defining the optimal conditions of sonication, liposomes containing tetracaine were prepared according to two experimental designs. At first, the drug was incorporated during the lipid film hydration, corresponding to the hydrophilic phase. In the second experimental design, liposomes were prepared with the drug incorporation during the lipid film preparation (drug in the lipophilic phase). In both cases, a RCCD experimental design was performed, having the lipid-cholesterol ratio and drug concentration as independent variables and the liposome size and encapsulation efficiency as dependent variables. The hydration solution was composed of a 60:40 ethanol/ water solution $(\%, v / v)$ for solubilizing the tetracaine at room temperature. The Table II shows the coded levels and actual values of the variables studied.

\section{Homogenization of vesicles size}

The samples obtained by the dry film hydration method were kept at rest for 30 minutes to reach 
TABLE I - Coded levels and actual values of the variables time and sonication power

\begin{tabular}{lccccc}
\hline \multirow{2}{*}{ Assays } & \multicolumn{2}{c}{ Time } & & \multicolumn{2}{c}{ Power } \\
\cline { 2 - 3 } & Coded & $\begin{array}{c}\text { Value } \\
\text { (min) }\end{array}$ & & $\begin{array}{c}\text { Coded } \\
\text { level }\end{array}$ & $\begin{array}{c}\text { Value } \\
\text { (kHz) }\end{array}$ \\
\hline 1 & -1.00 & 1.58 & & -1.00 & 11.4 \\
2 & +1.00 & 4.42 & & -1.00 & 11.4 \\
3 & -1.00 & 1.58 & & +1.00 & 18.4 \\
4 & +1.00 & 4.42 & & +1.00 & 18.4 \\
5 & -1.41 & 1.00 & & 0.00 & 14.9 \\
6 & +1.41 & 5.00 & & 0.00 & 14.9 \\
7 & 0.00 & 3.00 & & -1.41 & 10.0 \\
8 & 0.00 & 3.00 & & +1.41 & 19.8 \\
9 & 0.00 & 3.00 & & 0.00 & 14.9 \\
10 & 0.00 & 3.00 & & 0.00 & 14.9 \\
11 & 0.00 & 3.00 & 0.00 & 14.9 \\
\hline
\end{tabular}

TABLE II - Coded levels and actual values of the variables concentration of lipids: cholesterol and tetracaine concentration to the experiments performed in both hydrophilic and lipophilic phases

\begin{tabular}{lccccc}
\hline \multirow{2}{*}{ Assays } & \multicolumn{2}{c}{ Lipid:cholesterol ratio } & & \multicolumn{2}{c}{ Tetracaine } \\
\cline { 2 - 3 } \cline { 5 - 6 } & Coded level & $\begin{array}{c}\text { Value } \\
(\mathbf{m} \% / \mathbf{m} \%)\end{array}$ & & $\begin{array}{c}\text { Coded } \\
\text { level }\end{array}$ & $\begin{array}{c}\text { Value } \\
(\mathbf{m g} / \mathbf{m L})\end{array}$ \\
\hline 1 & -1.00 & $57: 43$ & & -1.00 & 2.5 \\
2 & -1.00 & $57: 43$ & & +1.00 & 8.5 \\
3 & +1.00 & $93: 7$ & & -1.00 & 2.5 \\
4 & +1.00 & $93: 7$ & & +1.00 & 8.5 \\
5 & 0.00 & $75: 25$ & & -1.41 & 1.0 \\
6 & 0.00 & $75: 25$ & & +1.41 & 10.0 \\
7 & -1.41 & $50: 50$ & & 0.00 & 5.5 \\
8 & +1.41 & $100: 0$ & & 0.00 & 5.5 \\
9 & 0.00 & $75: 25$ & & 0.00 & 5.5 \\
10 & 0.00 & $75: 25$ & & 0.00 & 5.5 \\
11 & 0.00 & $75: 25$ & & 0.00 & 5.5 \\
\hline$* 100 \%$
\end{tabular}

* $100 \%$ corresponds to a mass of $0.05 \mathrm{~g}$ in $10 \mathrm{~mL}$ of solvent

equilibrium before vesicles size homogenization. The liposome preparation was performed by sonication method using an ultrasonic sonicator (Unique ${ }^{\circledR}$ ) in the optimized conditions obtained in the experimental design ( $3 \mathrm{~min}$ and $16 \mathrm{KHz}$ ).

\section{Determination of the hydrodynamic radius and vesicles size distribution}

The hydrodynamic radius and liposomes size distribution were determined by the dynamic light scattering (DLS) using a laser (Malvern Zetasizer Nanoseries /NANO ZS90) in $1 \mathrm{~mL}$ of dispersion at $25^{\circ} \mathrm{C}$.

\section{Encapsulation efficiency (EE\%)}

Encapsulation efficiency was determined by ultracentrifugation (refrigerated centrifuge/Hermle $\mathrm{Z} 36 \mathrm{HK}$ ). The samples were centrifuged at $30,000 \mathrm{rpm}$ for $2 \mathrm{~h}$ at $10{ }^{\circ} \mathrm{C}$ and the supernatants diluted in 100 $\mathrm{mL}$ of a $60: 40$ ethanol/water $(\%, \mathrm{v} / \mathrm{v})$ to determine the concentration of non-encapsulated tetracaine. This technique was chosen based on the description in the literature that there is no difference between the method of dialysis through membranes and the ultracentrifugation method (López-Pinto, González-Rodríguez, Rabasco, 2005; Nasr et al., 2008). The encapsulation efficiency (EE\%) was determined by Equation 1:

$$
E E \%=\left[\frac{\text { Total Conc. TTc }- \text { non-encapsulated Conc. TTc }}{\text { Total Conc. TTc }}\right] \times 100
$$

where Total Conc.TTC is the initial concentration of tetracaine and non-encapsulated Conc. TTC is the tetracaine concentration in the supernatant.

\section{Determination of tetracaine concentration}

Initially, solutions containing tetracaine were prepared in 60:40 ethanol/water solution $(\%, \mathrm{v} / \mathrm{v})$ at different ranges of concentration $(0$ to $0.001 \mathrm{mg} / \mathrm{mL}$ and 0 to $0.015 \mathrm{mg} / \mathrm{mL}$, the latter being the maximum concentration that allowed a linear fit between the variables). From the experimental data were plotted standard curves of absorbance (analyzed by UV spectrophotometry at $310 \mathrm{~nm}$ ) versus concentration. All experiments were carried out in five repetitions. The tetracaine concentrations were determined in the supernatant after centrifugation (non-encapsulated drug).

\section{Stability study}

Stability study was performed with tetracaine-loaded liposomes stored at $4{ }^{\circ} \mathrm{C}$ over 15 days. Samples were taken at 0,7 and 15 days for measurement of drug loading as described at Encapsulation efficiency. Tetracaine content remaining in the liposome formulations were assessed by spectrophotometry as described in Determination of tetracaine concentration (Badran, Shalaby, Al-Omrani, 2012)

\section{Prediction models based on RCCD}

A central composite design (CCD) is an experimental design for building a second order (quadratic) model for the response variable. The CCD contains an imbedded 
factorial or fractional factorial design with center points that is augmented with a group of axial points ("star points') that allow estimation of curvature. If the distance from the center of the design space to a factorial point is \pm 1 unit for each factor, the distance from the center of the design space to a star point is $|\alpha|>1$. The factorial points contribute to the estimation of linear terms and interactions between factors and the center points provide information on the existence of curvature in the system. If the curve is found, the addition of the axial points allows to efficiently estimate the quadratic terms. In the rotatable $\mathrm{CCD}$ (RCCD), the $|\alpha|$ is 1.41 for two variables and describe a circular design geometry (Montgomery, 1997).

\section{Prediction models based on ANN}

The Multi-Layer Perceptron (MLP) trained with the Levenberg-Marquardt backpropagation algorithm is a technique that is widely used in applications based on artificial neural networks (Haykin, 1998; Levenberg, 1944; Marquardt, 1963). Figure 1 shows a three-layer MLP type network with $P$ neurons in the input layer, $K$ neurons in the hidden layer, and $H$ neurons in the output layer. The $i-t h$ neuron of the hidden yer processes the signal according to Equation 2:

$$
z_{i}=\varphi\left(\sum_{j=0}^{P-1} w_{i j}^{1} x_{j}\right)
$$

where $z_{i}(i=0,1, \ldots, K-1)$ is the output signal of the $i$-th neuron, $u_{j}(j=0,1, \ldots, P-1)$ is the $j$-th input signal, $w_{i j}^{1}$ are the weights associated with the connections between the input, $x_{i}$, and the output, $z_{i}$, and $\varphi(\cdot)$ is the activation function of the hidden layer. The output of the $h$-th neuron of the output layer is given by Equation 3:

$$
y_{h}=\phi\left(\sum_{i=0}^{K-1} w_{h i}^{2} z_{i}\right)
$$

where $y_{h}(h=0,1, \ldots, H-1)$ is the output signal of the $h$-th neuron, $w_{h i}^{2}$ are the weights associated with the connections between the output of the hidden layer, $z_{i}$, and the output of the output layer, $y_{h}$, and $\phi(\cdot)$ is the activation function of the output layer.

Thus, to improve the results of the analysis, an ANN type MLP trained with Levenberg Marquardt backpropagation algorithm was used. All experiments used two inputs ( $x_{1}$ and $\left.x_{2}\right)$, N neurons in the hidden layer, and one neuron in the output layer. The input and output data were normalized between zero and one in order to balance the training process (Zhang et al., 2010).

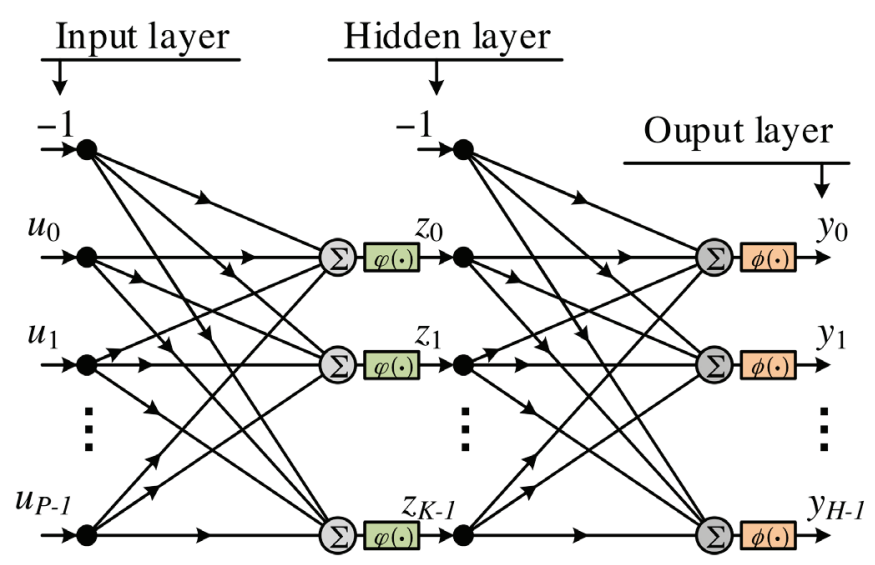

FIGURE 1 - The Multi-Layer perceptron with three-layer. The network with $P$ neurons in the input layer, $K$ neurons in the hidden layer, and $H$ neurons in the output layer.

\section{RESULTS AND DISCUSSION}

\section{Experimental design of sonication}

Figure 2 shows the response surface (A) and contour curve (B) for the variable mean diameter of liposomes containing $100 \%$ lipid $(0.05 \mathrm{~g} / 10 \mathrm{~mL})$. The values of power and sonication time corresponding to $16 \mathrm{kHz}$ and $3 \mathrm{~min}$, respectively, produce liposomes with a size smaller than $800 \mathrm{~nm}$. Thus, these sonication conditions were selected for further experiments due to decreasing the particles size that produced and desire.

The liposomes obtained in the LUV diameter were chosen for being able to extravasate blood into the interstitial space without causing pulmonary embolism (Lasic, 1993; Sapra, Allen, 2003). Furthermore, studies on the vesicles size show that the rate of recognition and removal by phagocytic defense system varies according to the diameter with a long half-life of liposomes $(>1000 \mathrm{~nm})$ of $0.2 \mathrm{~h}$ while the shortest have a $1.5 \mathrm{~h}$ halflife (Laverman et al., 1999).

Parameters as the power above $16 \mathrm{kHz}$ and times of sonication longer than 4.5 min during producing of liposomes led to obtaining samples with loss of physical stability, being observed the formation of viscous foam in suspension, resulting in a subsequent increase in vesicles diameter.

Figure $2 \mathrm{C}$ shows the Pareto diagram, in which we can observe that the quadratic term of the variable power of sonication and linear terms of the variables time and power $x$ time interaction were significant. The empirical model that describes the behavior of variables in the experimental conditions investigated is shown in Equation 4, with the significant terms in bold, where $D$ is the diameter, $t$ is time and $P$ power of sonication. 


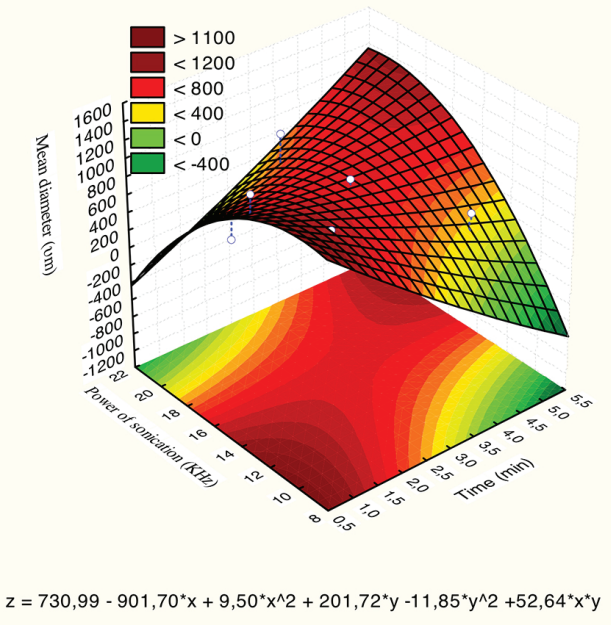

a)

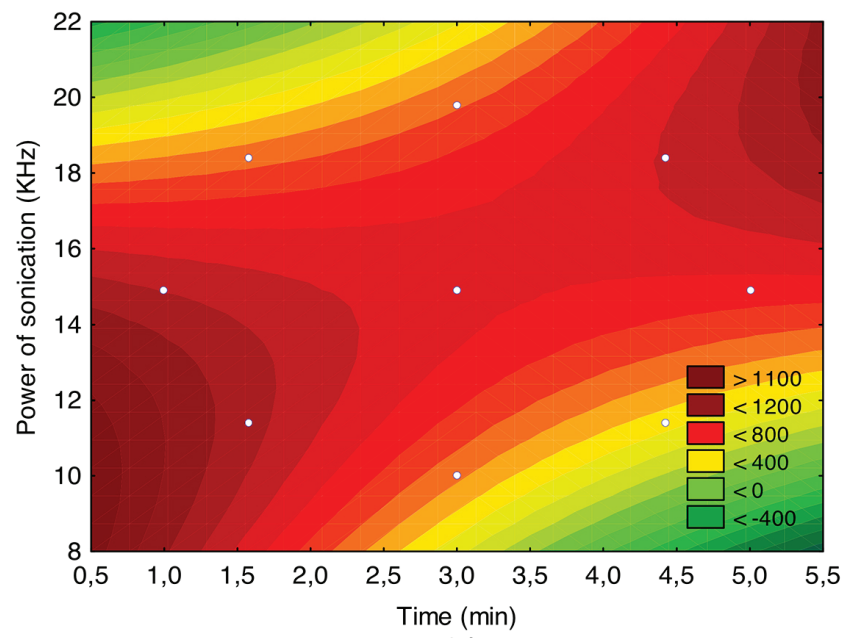

b)

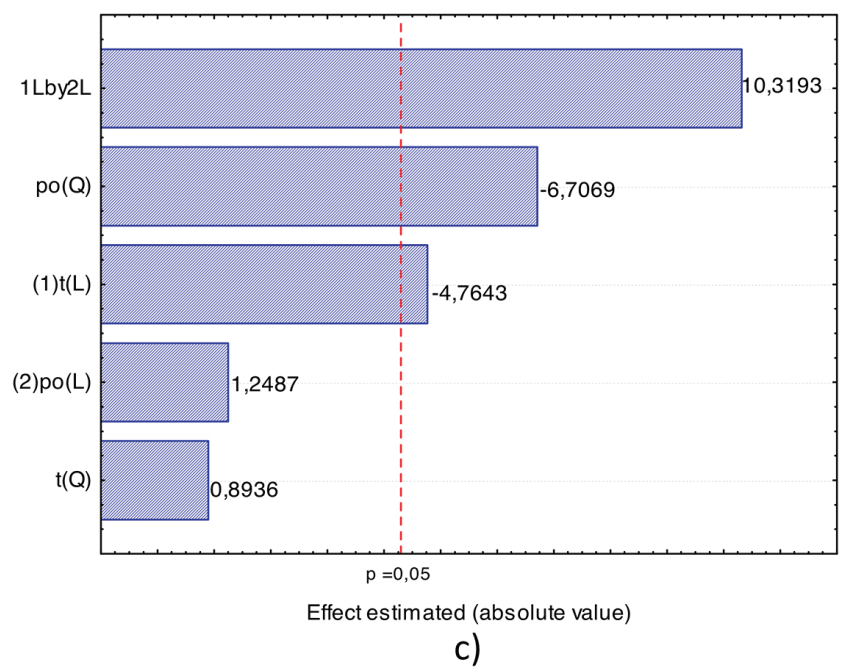

FIGURE 2 - Response surface (A), contour curve (B) and Pareto chart (C) for the mean diameter of liposomes depending on the power and sonication time (liposomes containing 100\% - $0.05 \mathrm{~g} / 10 \mathrm{~mL}$ soy phosphatidylcholine phospholipid).

$D=730.99-901.70 * t+9.50 * t^{2}+201.72 * P$

$-11.85 * P^{2}+52.64 * t * P$

\section{The effect of tetracaine-loaded on liposome stability}

The drug encapsulation over 15 days was performed in the liposomal formulations to verify the integrity of the vesicles, considering that was used a hydration solution of 60:40 ethanol/water solution (\%, v/v). The stability assays show that was not observed drug loss from the liposomes for the different formulations at $4{ }^{\circ} \mathrm{C}$ after 15 days of storage (data not shown). Ethanol at 40\%, v/v was used to dissolve tetracaine, on the other hand, could also dissolve phospholipid and cholesterol causing rupture of the liposomes, but it was not observed. Besides this, the presence of ethanol increases the permeability for topical application. According to Roberts and Water (Roberts, Walker, 1993), the use of $60 \%$ water increase hydration of the stratum corneum and enhances transdermal flux of a variety of drugs.

Mura and collaborators (2007) studied vesicles entrapping benzocaine prepared using 50:50 w/w phosphatidylcholine-cholesterol as the lipophilic phase and 50:50 v/v ethanol-water as the hydrophilic phase. Similarly, to this work, the addition of the drug into the hydrophilic phase enabled an improvement of entrapment efficiency.

\section{Experimental design - liposomes prepared in hydrophilic phase}

Figure 3A and 3B show, respectively, the response 
surface and the contour curve when the variable studied was the encapsulation efficiency of liposomes prepared in the hydrophilic phase. The optimal region for encapsulation efficiency was achieved. The critical values of the model that maximize the response were equivalent to a tetracaine concentration of $8.37 \mathrm{mg} / \mathrm{mL}$ and $79.5: 20.5$ lipid/cholesterol ratio $(\%, \mathrm{w} / \mathrm{w})$ for $39.7 \%$ maximum encapsulation efficiency.

Figure $3 \mathrm{C}$ shows, Pareto chart, that the only significant effect $(p<0.05)$ is the linear term of the variable tetracaine. This term is in bold in Equation 5, where $E$ represents the encapsulation efficiency, the TTc tetracaine concentration, and $\mathrm{Co}$ cholesterol content.

$$
\begin{aligned}
& E=-41.51+15.78 * T T c-0.83 * T T c^{2}+1.48 * C o \\
& -0.02 * C o^{2}-0.09 * T T C * C o
\end{aligned}
$$

In order to confirm the results predicted by the model, experiments were performed in triplicate under the optimum condition expected $(8.37 \mathrm{mg} / \mathrm{mL}$ and 79.5:20.5 lipid/cholesterol ratio $(\%, \mathrm{w} / \mathrm{w})$. Results are shown in TABLE II, yielding a mean value of $32.3 \pm 7.5 \%$, close to the value predicted by the model $(39.7 \%)$.

For being amphiphilic molecules, local anesthetics have a high affinity for cell membrane (Covino, 1986). The theories proposed to explain the mechanisms of action of these compounds can be classified into two categories. One that attributes the anesthetic effect to the binding on the voltage-dependent axons sodium channel protein. Another mechanism of action considers the interaction of local anesthetics with membrane lipids (known as the "lipid hypothesis") being responsible for the changes in sodium channel protein, and therefore to blockage in

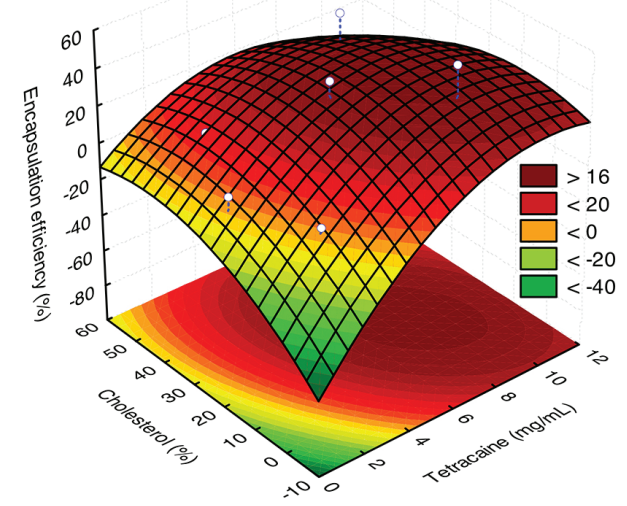

$z=-41,51+15,78^{*} x-0,83^{*} x^{\wedge} 2+1,48^{*} y-0,02^{*} y^{\wedge} 2-0,09^{*} x^{*} y$

a)

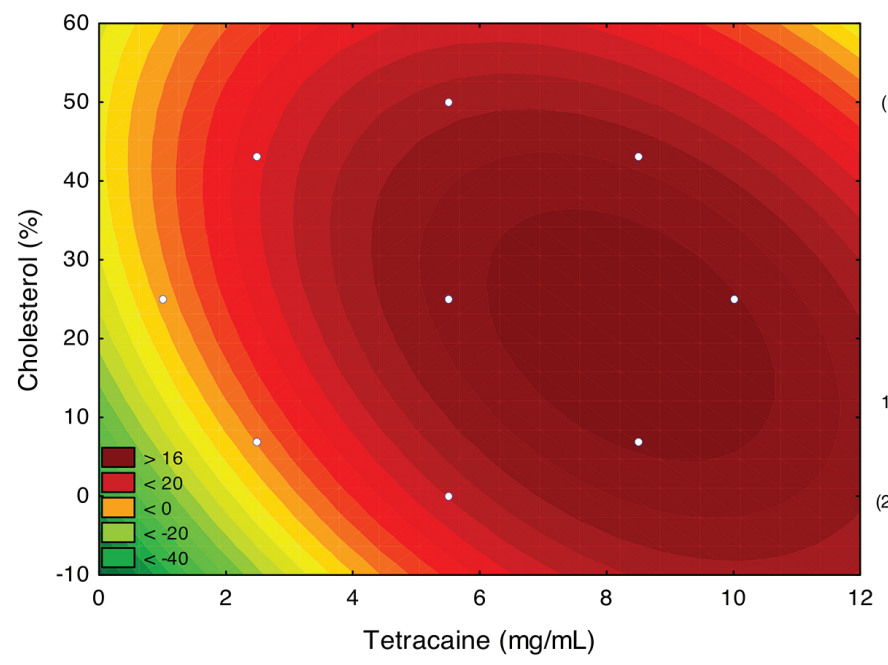

b)

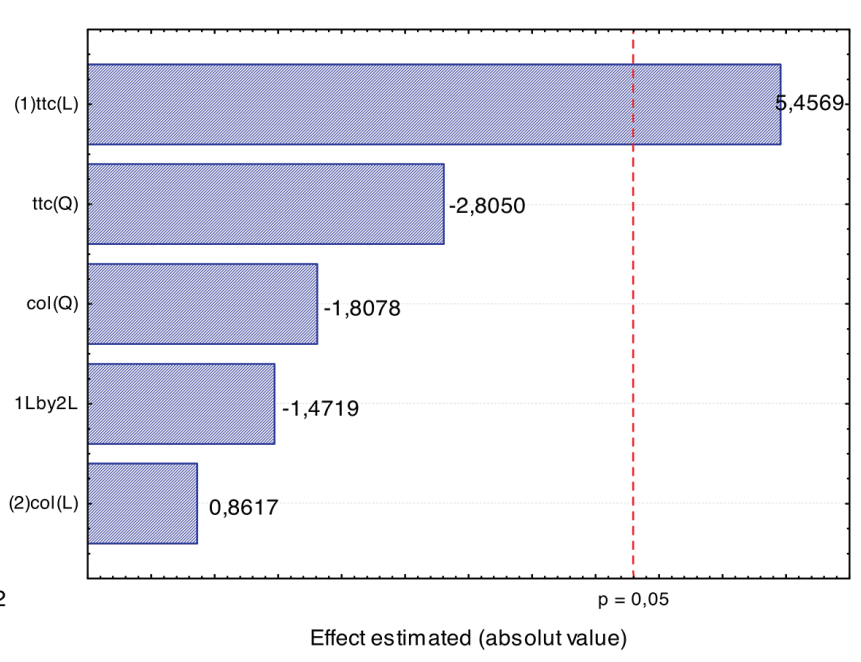

c)

FIGURE 3 - Response surface (A), contour curve (B) and Pareto chart (C) for the encapsulation efficiency of liposomes prepared in the hydrophilic phase. 
Comparative analyses of response surface methodology and artificial neural networks on incorporating tetracaine into liposomes

TABLE III - Results of experiments performed in triplicate using 79.5:20.5 lipid: cholesterol ratio (\%, w/w) and tetracaine concentration of $8.37 \mathrm{mg} / \mathrm{mL}$ for liposomes prepared in hydrophilic phase

\begin{tabular}{lccc}
\hline \multirow{2}{*}{ Assay } & Lipid:cholesterol ratio & Tetracaine & \multirow{2}{*}{ E $(\%)^{* *}$} \\
\cline { 2 - 3 } & Value $(\mathrm{m} \% / \mathrm{m} \%)^{*}$ & Value $(\mathrm{mg} / \mathrm{mL})$ & $32 \pm 3$ \\
2 & $79.5: 20.5$ & 8.37 & $25 \pm 5$ \\
3 & $79.5: 20.5$ & 8.37 & $40 \pm 4$ \\
\hline
\end{tabular}

* $100 \%$ corresponds to a mass of $0.05 \mathrm{~g}$ in $10 \mathrm{~mL}$ of solvent. **Maximum encapsulation efficiency predicted by model: $39.7 \%$

the nervous stimulus conduction. Despite these theories, studies have reported that tetracaine incorporated into phosphatidylcholine, phosphatidylserine and biological membranes was expelled from the bilayer by increasing pressure (Auger et al., 1987), an observation confirmed by the infrared technique.

The literature provides reports of the action of local anesthetics on the decrease of lipid phase transition temperature $(\mathrm{Tt})$ studied by different techniques. Studies using differential scanning calorimetry registered a $\mathrm{Tt}$ decrease of the phosphatidylcholine vesicles caused by the partition of benzocaine, procaine, tetracaine, lidocaine and dibucaine in concentrations equal to those required for $50 \%$ block in the nervous stimulus conduction (Lee, 1978).

De Paula and collaborators have studied the interaction of local anesthetics with lipid membranes using NMR, EPR and fluorescence techniques (de Paula, Schreier, 1996; Fraceto, de Paula, 2006). For amino-amide local anesthetics (lidocaine, mepivacaine, bupivacaine, and etidocaine) was demonstrated the differential effect of local anesthetics on the bilayer regions monitored by different paramagnetic probes. Besides, changes in lipids longitudinal relaxation times in the presence of local anesthetics and specific intermolecular interactions between the hydrogen of local anesthetics and liposomes provided evidence about their location within the bilayer (Fraceto, de Paula, 2006).

A fact that should be taken into consideration is that phosphate groups have negative charges, which can stabilize positive charges of local anesthetics. There are several reports in the literature that showed of local anesthetics competition with calcium ions by interaction with phosphate groups of lipid vesicles (de Paula, Schreier, 1996; Cullis, de Kruijff, 1978). This is the preferred pathway, i.e., the place where local anesthetics should meet most of the time. However, these amphiphilic molecules are spread quickly across the biological membrane and the aqueous phase (de Paula, Schreier, 1996) or are in dynamic balance, with preferential insertion within the bilayers. This location, determined by the local anesthetic interactions with phospholipids must be decisive for its the specific action (Ragsdale et al., 1994).

Figure 4A and 4B show, respectively, the response surface and contour curve for analysis of the mean diameter of liposomes. Liposomes type LUV with size $<1000 \mathrm{~nm}$, suitable for drug delivery system, were obtained for samples with 79.5:20.5 lipid: cholesterol ratio $(\%, \mathrm{w} / \mathrm{w})$ and the $8.37 \mathrm{mg} / \mathrm{ml}$ tetracaine concentration (optimal values according to Figure 4). Besides this, the Pareto chart (Figure 4C) shows the only significant effect is the interaction term between tetracaine and cholesterol concentration. This term is in bold in Equation 6.

$$
\begin{aligned}
& D=-488.64+306.94 * T T c-0.86 * T_{T}^{2}+46.69 * C o+ \\
& 0.52 * C^{2}-11.25 * \text { TTc } * \text { Co }
\end{aligned}
$$

The results showed the influence of the concentration of cholesterol and tetracaine on diameters of liposomes produced. High concentrations of tetracaine and low concentrations of cholesterol, as well as the inverse (low concentrations of tetracaine and high concentrations of cholesterol), favor the formation of liposomes with large sizes. Probably, this is due to the competition of these molecules (tetracaine or cholesterol) in the lipid bilayer at the time of liposomes formation. When both concentrations were increased, there was decrease in liposome diameter and decreased encapsulation efficiency.

\section{Experimental design - liposomes prepared in lipophilic phase}

Figure 5A and 5B show, respectively, the response surface and contour curve for encapsulation efficiency of liposomes prepared with tetracaine incorporated lipid film (lipid phase). Unlike the liposomes prepared with the drug incorporated during the lipid film hydration (or hydrophilic phase), there was no optimum value for efficiency, and the results varied between 5.6 and $83 \%$.

The highest value found was obtained for the lowest values of tetracaine $(1 \mathrm{mg} / \mathrm{mL})$ and cholesterol $(25 \%, \mathrm{w} / \mathrm{w})$ 


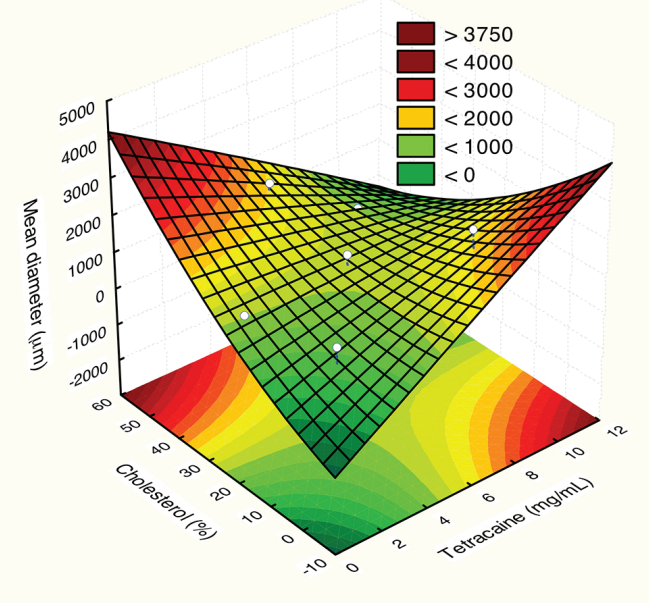

$z=-488,64+306,94^{*} x-0,86^{*} x^{\wedge} 2+46,69^{*} y+0,52^{*} y^{\wedge} 2-11,25^{*} x^{*} y$

a)

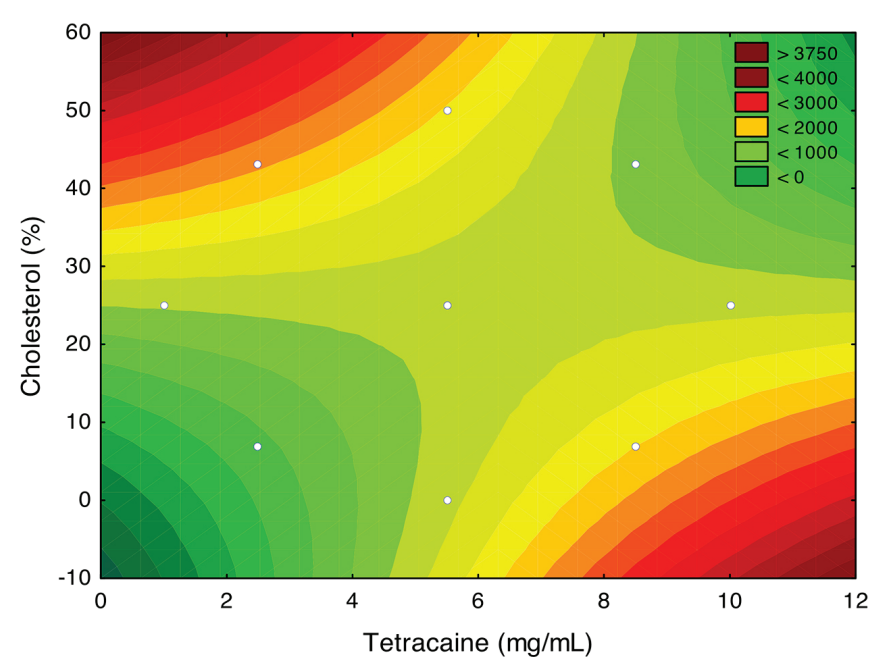

b)

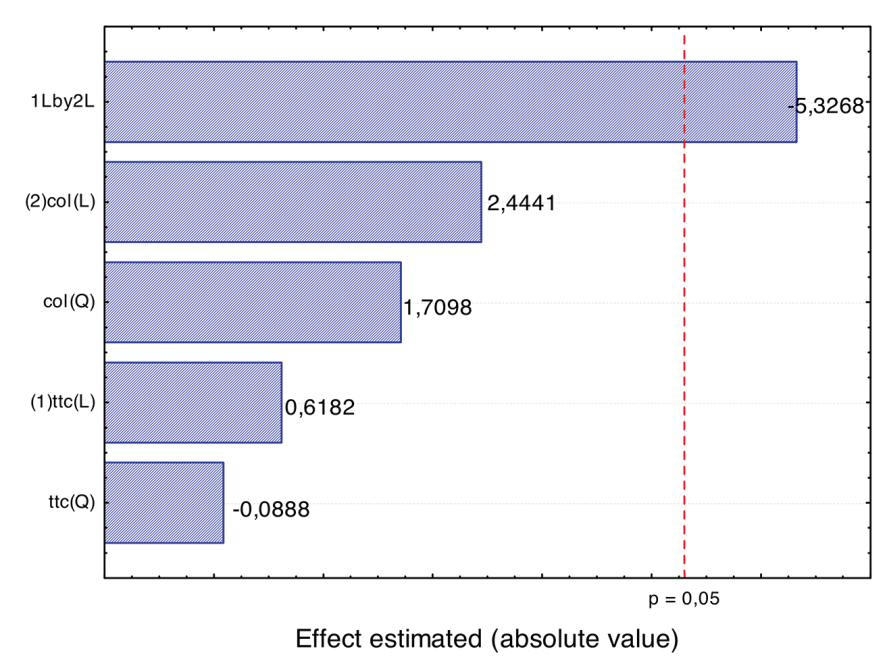

c)

FIGURE 4 - Response surface (A), contour curve (B) and Pareto chart (C) for the mean diameter of liposomes prepared in the hydrophilic phase.

concentration into the liposomal formulation, considered the drug content compared to the lipid content is low $(0.1: 75 \%, \mathrm{w} / \mathrm{w})$; values correspond a low rate of encapsulation not desired.

The Pareto chart (Figure $5 \mathrm{C}$ ) shows that the only effect that was not significant was the quadratic term of the variable cholesterol concentration. Equation 7 shows the theoretical model predicted, the significant terms of the model are in bold.

$$
\begin{aligned}
& E=50.51-9.19 * T T c+0.98 * T T c^{2}+0.56 * C o \\
& -0.00010 * C o^{2}-0.16 * T T c * C o
\end{aligned}
$$

The results demonstrate that despite the tetracaine being a lipophilic local anesthetic, the better drug encapsulation was achieved in the hydrophilic phase rather than in the lipophilic phase, as expected. Studies show that tetracaine incorporated into synthetic (phosphatidylcholine and phosphatidylserine) and biological membranes are expelled from the bilayer by pressure increase (Auger et al., 1987).

Skou (1954) and Seeman (1966) showed the effect of anesthetic in lipid phases. The results presented the local anesthetics incorporation by the lipid phase causes an expansion of the surface area of monolayers (Skou, 1954) and bilayers (Seeman, 1966). This expansion is favored by the difference between the anesthetic molecule length (shorter) and phospholipids. Thus, the anesthetic would create "void volume" below its insertion point, which would be offset by a conformational change of the 


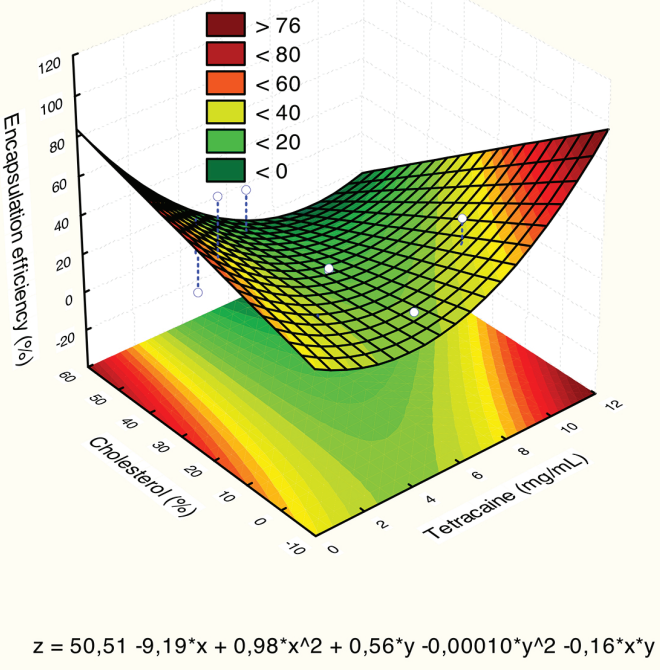

a)

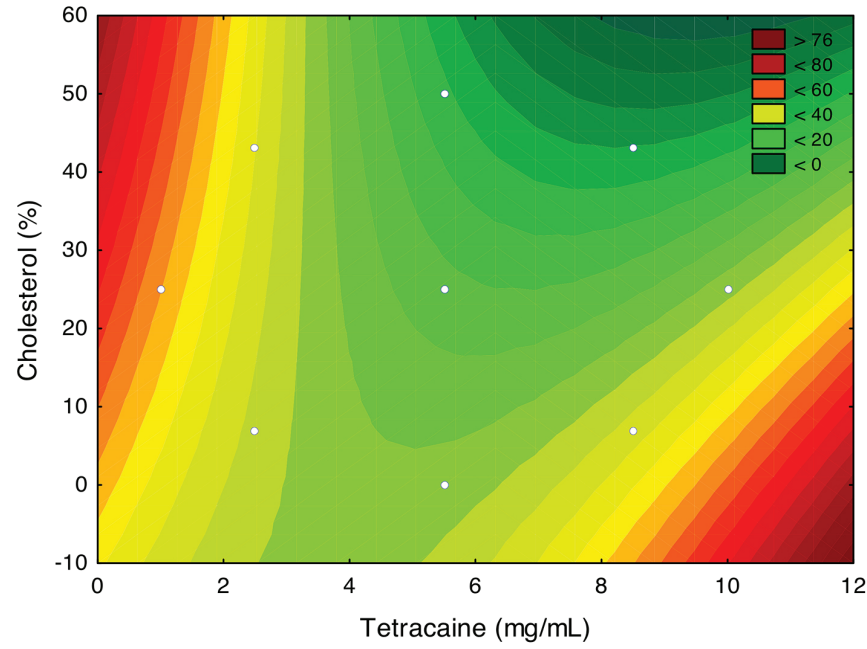

b)

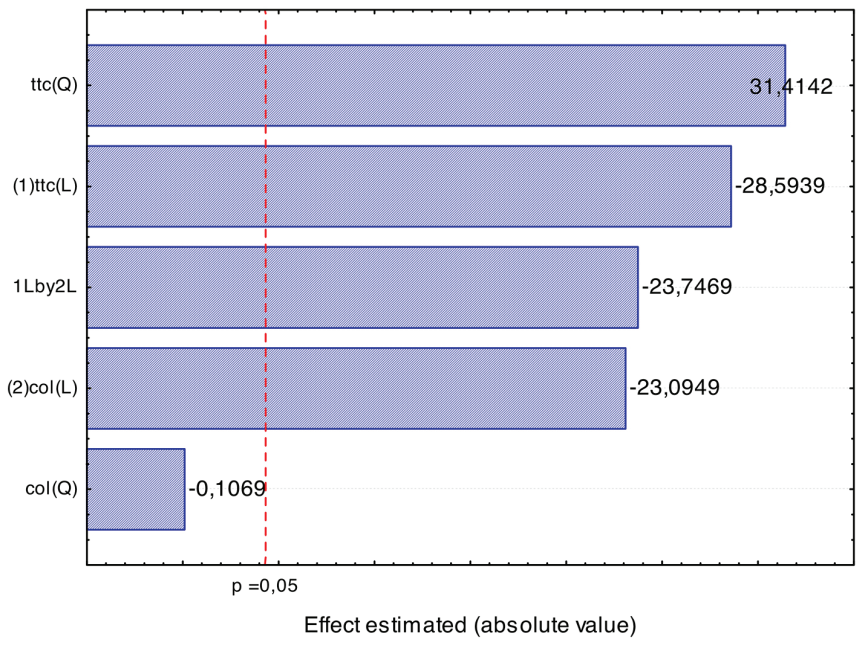

c)

FIGURE 5 - Response surface (A), contour curve (B) and Pareto chart (C) for the encapsulation efficiency of liposomes prepared in the lipophilic phase.

adjacent lipid chains decreasing the bilayer, as well as lateral expansion (Trudell, 1977).

\section{Experimental design with ANN}

All experimental design was modeled by Equations 4, 5, 6 and 7 were also adjusted by ANN. Tables IV, V, VI, and VII show the results obtained with ANN for equations $4,5,6$ and 7, respectively.

The ANN showed a smaller normalized error between the experimental value and the value obtained by the model (Takayama et al., 2003; Subramanian, Yajnik, Murthy, 2004; Zhang et al., 2010). The parameters found in the ANN are shown in Tables VIII, IX, X, and XI.
The results show that there is a substantial difference in accuracy of the ANN model compared to RSM-RCCD model (Tables IV-VII). This difference is caused due the RSM models work with regression, thus, need a specification as the linear polynomial function (second order or quadratic second order). According to Hanrahan and Lu (2006) and Ahmadi and Golian (2011), the number of terms of the polynomial is limited to points of Experimental Design. Furthermore, ANN-based models have good performance with no linear interpolation of data from different domains (Haykin, 1998).

Contrary to RSM-based methods, ANN need not specify a function, a priori, the interpolation process to act as universal approximators for any function, not only quadratic functions as the RSM-RCCD (Desai et al., 2008; 
TABLE IV - Comparison between experimental data and predictive models generated by RSM and ANN. Data associated with Equation 4

\begin{tabular}{lccc}
\hline \multirow{2}{*}{ Assays } & \multicolumn{3}{c}{ Diameter (nm) } \\
\cline { 2 - 4 } & Experimental & RSM & ANN \\
\hline 1 & 1039.0 & 1037.75 & 1039.0 \\
2 & 536.2 & 343.08 & 536.2 \\
3 & 210.0 & 560.08 & 210.0 \\
4 & 753.8 & 911.89 & 753.8 \\
5 & 1212.0 & 997.94 & 1212.0 \\
6 & 698.7 & 756.48 & 698.7 \\
7 & 351.7 & 522.79 & 351.7 \\
8 & 915.7 & 586.59 & 915.7 \\
9 & 809.4 & 839.21 & 807.72 \\
10 & 806.6 & 839.21 & 807.72 \\
11 & 895.8 & 839.21 & 807.72 \\
\hline Mean Normalized Error & $\mathbf{0 . 3 1 6 7}$ & $\mathbf{0 . 0 0 9 3}$ \\
\hline
\end{tabular}

TABLE V - Comparison between experimental data and predictive models generated by RSM and ANN. Data associated with Equation 5

\begin{tabular}{lccc}
\hline \multirow{2}{*}{ Assays } & \multicolumn{3}{c}{ Encapsulation efficiency (\%) } \\
\cline { 2 - 4 } & Experimental & RSM & ANN \\
\hline 1 & 11.0 & 9.74 & 11.0 \\
2 & 45.2 & 26.42 & 45.2 \\
3 & 0.0 & 0.56 & 0.0 \\
4 & 54.6 & 36.68 & 54.6 \\
5 & 3.8 & -4.31 & 3.8 \\
6 & 18.1 & 35.29 & 18.1 \\
7 & 21.6 & 19.42 & 21.6 \\
8 & 10.7 & 20.17 & 10.7 \\
9 & 28.8 & 32.30 & 33.4 \\
10 & 41.4 & 32.30 & 33.4 \\
11 & 30.1 & 32.30 & 33.4 \\
\hline Mean Normalized Error & $\mathbf{0 . 5 2 5 9}$ & $\mathbf{0 . 0 4 2 2}$ \\
\hline
\end{tabular}

Asiltürk, Çunkaş, 2011; Maran, Priya, 2015; Hanrahan, $\mathrm{Lu}, 2006)$.

The limitations of the techniques based on ANN are the quantity and quality of data used in its interpolation process or learning. However, the quality is more important than quantity. Some studies show that a sufficient amount of data for the ANN is equivalent to the number of experiments needed for a good regression process RSM. Other studies show that the design of experiments generates statistically
TABLE VI - Comparison between experimental data and predictive models generated by RSM and ANN. Data associated with Equation 6

\begin{tabular}{lccc}
\hline \multirow{2}{*}{ Assays } & \multicolumn{3}{c}{ Diameter $(\mathbf{n m})$} \\
\cline { 2 - 4 } & Experimental & RSM & ANN \\
\hline 1 & 1833 & 2033.11 & 1833.0 \\
2 & 887.1 & 915.49 & 887.1 \\
3 & 773.2 & 428.77 & 773.2 \\
4 & 2258 & 1741.15 & 2258.0 \\
5 & 1010 & 1028.44 & 1010.0 \\
6 & 924.9 & 1174.51 & 924.9 \\
7 & 1965 & 1714.27 & 1965.0 \\
8 & 615.5 & 1173.52 & 615.5 \\
9 & 968.7 & 1118.89 & 1140.3 \\
10 & 996.4 & 1118.89 & 1140.3 \\
11 & 1377 & 1118.89 & 1140.3 \\
\hline Mean Normalized Error & $\mathbf{0 . 2 3 6 7}$ & $\mathbf{0 . 0 4 4 9}$ \\
\hline
\end{tabular}

TABLE VII - Comparison between experimental data and predictive models generated by RSM and ANN. Data associated with Equation 7

\begin{tabular}{lccc}
\hline \multirow{2}{*}{ Assays } & \multicolumn{3}{c}{ Encapsulation efficiency (\%) } \\
\cline { 2 - 4 } & Experimental & RSM & ANN \\
\hline 1 & 5.60 & 40.36 & 5.62 \\
2 & 5.88 & 8.62 & 5.89 \\
3 & 16.0 & 34.78 & 16.00 \\
4 & 51.3 & 37.6 & 51.30 \\
5 & 83.00 & 52.24 & 83.00 \\
6 & 18.30 & 30.55 & 18.30 \\
7 & 35.09 & 13.36 & 35.06 \\
8 & 29.27 & 29.61 & 29.27 \\
9 & 21.09 & 21.55 & 21.88 \\
10 & 22.55 & 21.55 & 21.88 \\
11 & 22.00 & 21.55 & 21.88 \\
\hline Mean Normalized Error & $\mathbf{0 . 8 9 7 2}$ & $\mathbf{0 . 0 0 7 1}$ \\
\hline
\end{tabular}

well-distributed data in the input field, satisfying the data quality condition (Desai et al., 2008; Lou, Nakai, 2001). Thus, it can be concluded that the data used in RSM methods are sufficient for ANN training.

\section{CONCLUSIONS}

Considering the lack of information about liposomal formulations based on tetracaine, the use of experimental 
TABLE VIII - Parameters found for ANN associated to data in Table IV and Equation 4

\begin{tabular}{|c|c|c|c|c|c|c|c|c|}
\hline \multicolumn{9}{|c|}{ Input variables } \\
\hline & & Bias & & $x_{1}=t$ & & $x_{2}=P$ & & \\
\hline \multicolumn{9}{|c|}{ Neuron parameters of the input layer } \\
\hline & & $(j=0)$ & & $(j=1)$ & & $(j=2)$ & & \\
\hline$w_{1, j}^{1}$ & & -3.8716 & & 2.3586 & & -3.2581 & & \\
\hline$w_{2, j}^{1}$ & & 2.8818 & & -3.8989 & & -0.1912 & & \\
\hline$w_{3, j}^{1}$ & & -1.6871 & & 2.6345 & & 2.9997 & & \\
\hline$w_{4, j}^{1}$ & & -1.0675 & & 0.9035 & & -3.8350 & & \\
\hline$w_{5, j}^{1}$ & & 0.2269 & & 3.9071 & & -1.0901 & & \\
\hline$w_{6, j}^{1}$ & & 1.2557 & & 2.2130 & & 3.3801 & & \\
\hline$w_{7, j}^{1}$ & & -2.9486 & & -2.5385 & & 2.9185 & & \\
\hline$w_{8, j}^{1}$ & & 3.9108 & & 2.8963 & & -3.1234 & & \\
\hline \multicolumn{9}{|c|}{ Neuron parameters of the hidden layer } \\
\hline$w_{1,0}^{2}$ & $w_{1,1}^{2}$ & $w_{1,2}^{2}$ & $w_{1,3}^{2}$ & $w_{1,4}^{2}$ & $w_{1,5}^{2}$ & $w_{1,6}^{2}$ & $w_{1,7}^{2}$ & $w_{1,8}^{2}$ \\
\hline-0.5588 & -3.8716 & 2.8818 & -1.6871 & -1.0675 & 0.2269 & 1.2557 & -2.9486 & 3.9108 \\
\hline
\end{tabular}

TABLE IX - Parameters found for ANN associated to data in Table V and Equation 5

\begin{tabular}{|c|c|c|c|c|c|c|c|c|c|c|}
\hline \multicolumn{11}{|c|}{ Input variables } \\
\hline & & Bias & & & $x_{1}=T T c$ & & & $x_{2}=C o$ & & \\
\hline \multicolumn{11}{|c|}{ Neuron parameters of the input layer } \\
\hline & & $(j=0)$ & & & $(j=1)$ & & & $(j=2)$ & & \\
\hline$w_{1, j}^{1}$ & & 4.4071 & & & -1.9592 & & & 3.9894 & & \\
\hline$w_{2, j}^{1}$ & & 3.5336 & & & -3.0959 & & & -3.1337 & & \\
\hline$w_{3, j}^{1}$ & & 2.2213 & & & -2.7661 & & & -3.6703 & & \\
\hline$w_{4, j}^{1}$ & & 1.5015 & & & -0.0871 & & & -4.4017 & & \\
\hline$w_{5, j}^{1}$ & & -0.4460 & & & 0.3748 & & & -4.4183 & & \\
\hline$w_{6, j}^{1}$ & & 0.5677 & & & 4.1302 & & & 1.3483 & & \\
\hline$w_{7, j}^{1}$ & & -1.2688 & & & -0.8729 & & & 4.4473 & & \\
\hline$w_{8, j}^{1}$ & & -2.2461 & & & -0.7728 & & & -4.4547 & & \\
\hline$w_{9, j}^{1}$ & & 3.3439 & & & 3.5850 & & & -2.5824 & & \\
\hline$w_{10, j}^{1}$ & & 4.6356 & & & 0.9081 & & & 4.0700 & & \\
\hline \multicolumn{11}{|c|}{ Neuron parameters of the hidden layer } \\
\hline$w_{1,0}^{2}$ & $w_{1,1}^{2}$ & $w_{1,2}^{2}$ & $w_{1,3}^{2}$ & $w_{1,4}^{2}$ & $w_{1,5}^{2}$ & $w_{1,6}^{2}$ & $w_{1,7}^{2}$ & $w_{1,8}^{2}$ & $w_{1,9}^{2}$ & $w_{1,10}^{2}$ \\
\hline-0.183 & -0.502 & -0.883 & 0.945 & 0.100 & -0.107 & 0.793 & 0.323 & 0.143 & 0.082 & 0.660 \\
\hline
\end{tabular}


TABLE X - Parameters found for ANN associated to data in Table VI and Equation 6

\begin{tabular}{|c|c|c|c|c|c|c|c|c|c|c|}
\hline \multicolumn{11}{|c|}{ Input variables } \\
\hline & & Bias & & & $x_{1}=T T c$ & & & $x_{2}=C o$ & & \\
\hline \multicolumn{11}{|c|}{ Neuron parameters of the input layer } \\
\hline & & $(j=0)$ & & & $(j=1)$ & & & $(j=2)$ & & \\
\hline$w_{1, j}^{1}$ & & -4.3503 & & & 1.5599 & & & 4.2188 & & \\
\hline$w_{2, j}^{1}$ & & -3.4715 & & & 3.6218 & & & 2.5979 & & \\
\hline$w_{3, j}^{1}$ & & 2.5825 & & & -0.5429 & & & -4.3068 & & \\
\hline$w_{4, j}^{1}$ & & -1.4577 & & & 4.3456 & & & 0.5625 & & \\
\hline$w_{5, j}^{1}$ & & -0.5522 & & & 3.1211 & & & -3.1371 & & \\
\hline$w_{6, j}^{1}$ & & -0.4053 & & & -1.5376 & & & -4.0681 & & \\
\hline$w_{7, j}^{1}$ & & 1.2791 & & & 2.1307 & & & 3.9591 & & \\
\hline$w_{8, j}^{1}$ & & 2.5696 & & & 3.3058 & & & -2.806 & & \\
\hline$w_{9, j}^{1}$ & & -3.4682 & & & -2.8528 & & & 3.3638 & & \\
\hline$w_{10, j}^{1}$ & & -4.2272 & & & -0.1236 & & & 4.6009 & & \\
\hline \multicolumn{11}{|c|}{ Neuron parameters of the hidden layer } \\
\hline$w_{1,0}^{2}$ & $w_{1,1}^{2}$ & $w_{1,2}^{2}$ & $w_{1,3}^{2}$ & $w_{1,4}^{2}$ & $w_{1,5}^{2}$ & $w_{1,6}^{2}$ & $w_{1,7}^{2}$ & $w_{1,8}^{2}$ & $w_{1,9}^{2}$ & $w_{1,10}^{2}$ \\
\hline-0.168 & 0.201 & -1.494 & -0.169 & 0.968 & -0.169 & 0.334 & 0.424 & 0.065 & 0.480 & 0.351 \\
\hline
\end{tabular}

TABLE XI - Parameters found for ANN associated to data in Table VII and Equation 7

\begin{tabular}{|c|c|c|c|c|c|c|c|c|}
\hline \multicolumn{9}{|c|}{ Input variables } \\
\hline & & Bias & & $x_{1}=T T c$ & & $x_{2}=C o$ & & \\
\hline \multicolumn{9}{|c|}{ Neuron parameters of the input layer } \\
\hline & & $(j=0)$ & & $(j=1)$ & & $(j=2)$ & & \\
\hline$w_{1, j}^{1}$ & & -4.4248 & & 1.0770 & & 3.4484 & & \\
\hline$w_{2, j}^{1}$ & & -2.7884 & & 2.1154 & & -3.4078 & & \\
\hline$w_{3, j}^{1}$ & & -1.7371 & & 2.0552 & & 3.3694 & & \\
\hline$w_{4, j}^{1}$ & & -1.2357 & & 3.2373 & & 2.3255 & & \\
\hline$w_{5, j}^{1}$ & & 0.1457 & & -3.4256 & & 2.0736 & & \\
\hline$w_{6, j}^{1}$ & & -1.5048 & & -3.4734 & & 2.3872 & & \\
\hline$w_{7, j}^{1}$ & & 3.2544 & & 2.5771 & & -2.9282 & & \\
\hline$w_{8, j}^{1}$ & & -3.7784 & & -0.6764 & & -4.0875 & & \\
\hline \multicolumn{9}{|c|}{ Neuron parameters of the hidden layer } \\
\hline$w_{1,0}^{2}$ & $w_{1,1}^{2}$ & $w_{1,2}^{2}$ & $w_{1,3}^{2}$ & $w_{1,4}^{2}$ & $w_{1,5}^{2}$ & $w_{1,6}^{2}$ & $w_{1,7}^{2}$ & $w_{1,8}^{2}$ \\
\hline-0.2297 & 1.2364 & 0.3094 & -0.4297 & 0.0452 & -0.3109 & 1.3494 & 1.6949 & -0.4061 \\
\hline
\end{tabular}


planning tool was useful in order to optimize the preparing conditions for liposomes containing this drug. It was possible to determine the optimum conditions for sonication of liposomes containing 100\% lipids for obtaining LUV liposomes, these conditions were used to prepare vesicles containing the drug. According to the results of this study, it was found that the drug encapsulation efficiency was higher in the hydrophilic phase, despite tetracaine being a hydrophobic anesthetic. A comparative analysis between the RCCD-RSM and ANN techniques in process modeling and prediction surfaces was performed. The experimental results were compared using the Normalized Mean Error as used in the literature. In all cases analyzed, the ANN obtained more refined prediction errors that RCCD-RSM technique, showing up as a viable alternative for characterizing surfaces and, in order to identify the relevant parameters which, lead to an optimization of liposomes with tetracaine for more effective local anesthesia.

\section{ACKNOWLEDGMENTS}

Financial support for this research was provided from Pró-reitoria para Assuntos de Pesquisa Pósgraduação (Propesq/UFPE). We are grateful to Department of Physics from Federal University of Pernambuco for the access to the DLS facility.

\section{FUNDING}

This work was supported by the Pró-reitoria para Assuntos de Pesquisa e Pós-graduação (Propesq/UFPE).

\section{CONFLICT OF INTEREST}

The author(s) declare(s) that they have no declaration of interests to disclose.

\section{REFERENCES}

Ahmadi H, Golian A. Response surface and neural network models for performance of broiler chicks fed diets varying in digestible protein and critical amino acids from 11 to 17 days of age. Poultry Sci. 2011;90(9):2085-2096.

Asiltürk İ, Çunkaş M. Modeling and prediction of surface roughness in turning operations using artificial neural network and multiple regression method. Expert Syst Appl. 2011;38(5):5826-5832.
Auger M, Jarrell HC, Smith IC, Wong PT, Siminovitch DJ, Mantsch HH. Pressure-induced exclusion of a local anesthetic from model and nerve membranes. Biochemistry 1987;26:85138516.

Badran M, Shalaby K, Al-Omrani A. Influence of the flexible liposomes on the skin deposition of a hydrophilic model drug, carboxyfluorescein: dependency on their composition. ScientificWorldJournal. 2012;2012:134876.

Covino BG. Pharmacology of local anaesthetic agents. Brit J Anaesth. 1986;58(7):701-16.

Cullis PR, de Kruijff B. Polymorphic phase behaviour of lipid mixtures as detected by 31P NMR. Evidence that cholesterol may destabilize bilayer structure in membrane systems containing phosphatidylethanolamine. Biochim Biophys Acta 1978;507(2):207-218.

de Paula E, Schreier S. Molecular and physicochemical aspects of local anesthetic-membrane interaction. Braz J Med Biol Res. 1996;29(7):877-94.

Desai KM, Survase SA, Saudagar PS, Lele SS, Singhal RS. Comparison of artificial neural network (ANN) and response surface methodology (RSM) in fermentation media optimization: Case study of fermentative production of scleroglucan. Biochem Eng J. 2008;41(3):266-273.

El Maghraby GM, Arafa MF, Essa EA, Vesicular nanostructures for transdermal delivery. In Applications of Nanocomposite Materials in Drug Delivery. Woodhead Publishing Series in Biomaterials, p 469-490, 2018.

Fisher R, Murphy M, Hung O, Mezei M, Stewart R. Absorption of liposome-encapsulated tetracaine versus nonliposomeencapsulated tetracaine from open wounds in rabbits. Am J Emerg Med. 1994;12(5):521-523.

Foldvari M, Jarvis B, Oguejiofor CJN. Topical dosage form of liposomal tetracaine: effect of additives on the in vitro release and in vivo efficacy. J Control Release 1993;21(3):193-205.

Fraceto LF, de Paula E. Interação de anestésicos locais com lipossomas determinada por espectroscopia de infravermelho. Rev Ciênc Farm Básica Apl. 2006;27(1):27-35.

Friedmann NK, Juliano RL. Liposome encapsulated tetracaine lowers blood glucose. Biochim Biophys Acta 1984;799(2):195198. 
Hanrahan G, Lu K. Application of factorial and response surface methodology in modern experimental design and optimization. Crit Rev Anal Chem. 2006;36(3-4):141-151.

Haykin S. Neural networks: A comprehensive foundation. New Jersey: Prentice Hall PTR; 1998.

Lasic DD. Liposomes: from physics to applications. New York: Elsevier, 1993. 575 p.

Laverman P, Boerman OC, Oyen WJ, Dams ET, Storm G, Corstens FH. Liposomes for scintigraphic detection of infection and inflammation. Adv Drug Deliv Rev. 1999;37(1-3):225-235.

Lee AG. Effects of charged drugs on the phase transition temperatures of phospholipid bilayers. Biochim Biophys Acta. 1978;514(1):95-104.

Levenberg K. A method for the solution of certain non-linear problems in least squares. Quart Appl Math. 1944;2(2):164-168.

López-Pinto JM, González-Rodríguez ML, Rabasco AM. Effect of cholesterol and ethanol on dermal delivery from DPPC liposomes. Int J Pharm. 2005;298(1):1-12.

Lou W, Nakai S. Artificial neural network-based predictive model for bacterial growth in a simulated medium of modifiedatmosphere-packed cooked meat products. Agr Food Chem. 2001;49(4):1799-1804.

Maran PM, Priya B. Comparison of response surface methodology and artificial neural network approach towards efficient ultrasound-assisted biodiesel production from muskmelon oil. Ultrason Sonochem. 2015;23:192-200.

Marquardt DW. An algorithm for least-squares estimation of nonlinear parameters. SIAM J Appl Math. 1963;11(2):431-441.

Moghaddam MG, Khajeh M. Comparison of response surface methodology and artificial neural network in predicting the microwave-assisted extraction procedure to determine zinc in fish muscles. Food Nut Sci. 2011;2(8):803-808.

Montgomery DC. Design and analysis of experiments. New York: Wiley; 1997.704 p.

Moussa HG, Husseini GA, Abel-Jabbar N, Ahmad SE. Use of model predictive control and artificial neural networks to optimize the ultrasonic release of a model drug from liposomes. IEEE Trans Nanobiosci. 2017;16(3):149-156.
Mura PM, Maestrelli F, González-Rodríguez ML, Michelacci I, Ghelardini C, Rabasco AM. Development, characterization and in vivo evaluation of benzocaine-loaded liposomes. Eur J Pharm Biopharm. 2007;67(1):86-95.

Nasr M, Mansour S, Mortada ND, Elshamy AA. Vesicular aceclofenac systems: a comparative study between liposomes and niosomes. J Microencapsul. 2008;25(7):499-512.

Ragsdale DS, McPhee JC, Scheuer T, Catterall WA. Molecular determinants of state-dependent block of $\mathrm{Na}+$ channels by local anesthetics. Science. 1994;265(5179):1724-1728.

New RRC. Liposomes: a practical approach. Oxford: Oxford University Press; 1990. 301 p.

Roberts MS, Walker M. Water: the most natural penetration enhancer. In: Walters K, Hadgraft J, editors. Pharmaceutical skin penetration enhancement. New York: Marcel Dekker; 1993. p. 1-30.

Sapra P, Allen TM. Ligand-targeted liposomal anticancer drugs. Prog Lipid Res. 2003;42(5):439-62.

Seeman P. Erythrocyte membrane stabilization by steroids and alcohols; a possible model for anesthesia. Biochem Pharmacol. 1966;15(10):1632-1637.

Siepmann J, Siepmann F. Mathematical modeling of drug delivery. Int J Pharm. 2008;364(2):328-343.

Skou JC. Local anaesthetics. V. The action of local anaesthetics on monomolecular layers of stearic acid. Acta Pharmacol Toxicol. (Copenh) 1954;10(4):317-324.

Subramanian N, Yajnik A, Murthy RSR. Artificial neural network as an alternative to multiple regression analysis in optimizing formulation parameters of cytarabine liposomes. AAPS Pharm Sci Tech. 2004;5(1):E4.

Sun Y, Peng Y, Chen Y, Shukla AJ. Application of artificial neural networks in the design of controlled release drug delivery systems. Adv Drug Deliv Rev. 2003;55(9):1201-1215.

Sulaiman ISC, Basri M, Masoumi HRF, Ashari SE, Basri H, Ismail M. Predicting the optimum compositions of a transdermal nanoemulsion system containing an extract of Clinacanthus nutans leaves (L.) for skin antiaging by artificial neural network model. J Chemom. 2017;31(7):e2894. 
Takayama K, Fujikawa M, Nagai T. Artificial neural network as a novel method to optimize pharmaceutical formulations. Pharm Res. 1991;16(1):1-6.

Takayama K, Fujikawa M, Obata Y, Morishita M. Neural network-based optimization of drug formulations. Adv Drug Deliv Rev. 2003;55(9):1217-1231.

Trudell JR. A unitary theory of anesthesia based on lateral phase separations in nerve membranes. Anesthesiology. 1977;46(1):510.
Zhang Y, Xu J, Yuan Z, Xu H, Yu Q. Artificial neural networkgenetic algorithm-based optimization for the immobilization of cellulase on the smart polymer Eudragit L-100. Bioresour Technol. 2010;101(9):3153-3158.

Received for publication on $29^{\text {th }}$ May 2018 Accepted for publication on $24^{\text {th }}$ September 2018 